\title{
Supplémentation alimentaire pour synchroniser les chaleurs et améliorer les performances de reproduction des caprins à Madagascar
}

\author{
Julien Honoré Andrianarisoa ${ }^{1}$ \\ Josoa Ramarolanonana Randriamalala 1,2* \\ Arsène Jules Mbolatianarizao Randrianariveloseheno ${ }^{2}$ \\ Radobarimanjaka Rabeniala ${ }^{1}$
}

Mots-clés

Caprin, fertilité, prolificité, flushing, Madagascar

Submitted: 20 June 2019

Accepted: 24 April 2020

Published: 29 June 2020

DOI: 10.19182/remvt.31877

\section{Résumé}

Les zones littorales du Sud-Ouest malgache sont les plus arides de l'île. L'élevage de petits ruminants y est la principale source de revenus pour les populations locales. Or, leur reproduction, surtout celle des caprins, y est entravée par des conditions défavorables zootechniques (génétique et conduite d'élevage) et agroclimatiques (faible disponibilité fourragère pendant la longue saison sèche). Des méthodes d'amélioration des performances de la reproduction de l'élevage caprin existent : I'insémination artificielle, les traitements hormonaux, l'effet mâle et la supplémentation alimentaire ou flushing. Le flushing est une méthode simple à mettre œuvre et n'implique pas de modification importante des pratiques pastorales ; il est le plus adapté aux conditions de ce site semi-aride. Deux lots d'animaux déparasités ont été utilisés durant 45 jours en juillet-août (saison sèche) : un lot de 207 femelles avec un aliment énergétique (flushing), du manioc sec, à raison de 500 g/jour, et un lot témoin de 184 chèvres. Des paramètres de reproduction ont été enregistrés : taux de fertilité, taux de fécondité, taux de prolificité et taux de survie à l'âge de 30 jours. Des améliorations significatives de la fertilité ( $81 \%$ vs $28 \%$ ) et de la fécondité ( $69 \%$ vs $25 \%$ ) des femelles traitées ont été observées. La prolificité du lot traité (120\%) a été légèrement supérieure à celle du lot témoin $(115 \%)$, sans différence significative. Trente jours après la naissance, le taux de survie des chevreaux du lot traité a été significativement plus élevé (67\% vs $25 \%$ pour le lot témoin). La méthode du flushing a amélioré les paramètres de reproduction des caprins autochtones ainsi que la productivité numérique et économique de l'élevage caprin en zone semi-aride du Sud-Ouest malgache.

- Comment citer cet article : Andrianarisoa J.H., Randriamalala J.R., Mbolatianarizao Randrianariveloseheno A.J., Rabeniala R., 2020. Feed supplementation to synchronize estrous and improve the reproductive performance of goats in Madagascar. Rev. Elev. Med. Vet. Pays Trop., 73 (2): 99-106, doi : $10.19182 /$ remvt.31877

\footnotetext{
1. Diagnostic environnemental et recherches appliquées pour le développement en milieu rural (DERAD), Vontovorona, Antananarivo, Madagascar.

2. Ecole supérieure des sciences agronomiques (ESSA), BP 175,

Université d'Antananarivo, Ankatso, Antananarivo 101, Madagascar.

* Auteur pour la correspondance

Email : ramarolanonana@gmail.com
}

\section{INTRODUCTION}

Lélevage de petits ruminants de races locales (caprins, Capra hircus, et ovins, Ovis aries) est principalement pratiqué dans l'extrême Sud et le Sud-Ouest malgache (régions Atsimo Andrefana et Androy) où se concentre plus de $80 \%$ du cheptel national (ministère de l'Elevage, 2012). L'élevage caprin y est la principale source de revenus pour les populations vivant dans les zones côtières, les plus arides 
de l'île (Rabeniala et al., 2009 ; Raoliarivelo et al., 2010). Les raisons suivantes peuvent expliquer ce fait : a) la zone est globalement peu propice à l'agriculture car trop aride, les revenus provenant des ventes régulières de caprins assurent l'achat de produits de première nécessité (Raoliarivelo et al., 2010 ; Hanke, 2016) ; b) les caprins s'adaptent bien au climat semi-aride qui y prévaut ; et c) le cheptel, qui constitue une source importante de revenus des ménages, est considéré comme un signe extérieur de richesse (Raoliarivelo et al., 2010). Les caprins sont également des animaux prolifiques car une femelle peut donner naissance jusqu'à trois individus par portée (Amoah et al., 1996 ; Kusina et al., 2001) et deux à trois portées en deux ans. De plus, l'élevage caprin peut également être considéré comme une activité alternative à la fabrication de charbon de bois qui est la principale source de dégradation des fourrés xérophiles du SudOuest malgache (Randriamalala et al., 2016 ; 2017). En effet, les deux activités (élevage et fabrication de charbon) exploitent les fourrés xérophiles qui servent de pâturage aux caprins et de sources de bois à carboniser.

L'élevage caprin peut ainsi servir de levier pour lutter contre l'insécurité alimentaire et la pauvreté, phénomènes qui prévalent dans le grand Sud malgache. Mais les conduites actuelles de cet élevage dans cette région, qui ne permettent d'obtenir qu'une mise bas par an, limitent sa production pendant la courte saison des pluies (Andrianarisoa, 2013 ; Feldt et al., 2016). De plus, cette mise bas a surtout lieu pendant la saison sèche (juin-juillet; Andrianarisoa, 2013) au cours de laquelle une faible disponibilité fourragère prévaut dans les pâturages (Rabeniala et al., 2009), d'où l'intérêt de l'améliorer. L'induction des chaleurs des femelles en dehors de la saison de reproduction naturelle est possible et peut augmenter le nombre de naissances et améliorer les performances de reproduction en général. Durant la période de déficit fourrager, cette induction des chaleurs après mise bas aboutit à un groupage des naissances en saison des pluies (contre-saison). Deux moyens sont possibles pour induire et grouper les chaleurs en contre-saison. Le premier consiste à utiliser des produits hormonaux (Greyling et Van der Nest, 2000 ; Motlomelo et al., 2002 ; Lehloenya et al., 2008) combinés avec l'insémination artificielle (Lehloenya et al., 2005 ; 2008) ou avec la monte naturelle (Chemineau, 1989 ; Kausar et al., 2009). Le second consiste à utiliser des moyens zootechniques (photopériodisme, effet mâle, et supplémentation alimentaire ou flushing) combinés avec la monte naturelle (Acero-Camelo et al., 2008). L'application des moyens hormonaux et l'insémination artificielle sont coûteuses et requièrent une maîtrise de la technologie assez complexe (stockage et administration des produits hormonaux et des semences) qui n'est pas à la portée des éleveurs du grand Sud malgache. D'où la nécessité de trouver des techniques plus simples et plus pratiques pour grouper les chaleurs en contre-saison.

Le flushing peut être envisagé (Molle et al., 1995 ; 1997 ; Kusina et al., 2001). Notre objectif était de tester avec des éleveurs pilotes l'efficacité de cette technique pour induire les chaleurs de caprins dans le Sud-Ouest malgache en saison sèche et améliorer les performances de reproduction (fertilité, fécondité et prolificité).

\section{MATERIEL ET METHODES}

\section{Site d'étude}

Le site d'étude inclut les communes rurales de Soalara Sud et de Beheloke, dans le district de Toliara II, dans la région Atsimo Andrefana, du Sud-Ouest malgache. Le climat est de type semi-aride avec une pluviométrie annuelle moyenne de 350 millimètres et une saison sèche d'environ neuf mois (avril à décembre ; Gaspard et al., 2018). Les sols sont de nature sableuse peu évoluée dans les formations dunaires, ou de nature calcaire sur affleurements de grès calcaires. La végétation naturelle est un fourré xérophile à Didiereaceae et Euphorbiaceae (Cornet et Guillaumet, 1976).

Les populations locales vivent de l'agriculture, de l'élevage de petits ruminants, de la pêche et de la fabrication de charbon de bois (Raoliarivelo et al., 2010). Le maïs, le manioc et la patate douce sont les principales cultures (Rabeniala et al., 2009). L'élevage des petits ruminants est la principale source de revenus (Raoliarivelo et al., 2010). L'objectif est de posséder le plus de têtes de bétail possible. La densité des petits ruminants dans le site d'étude est environ d'une tête par hectare et chaque ménage possède en moyenne 60 têtes (de 2 à 180 têtes ; Rabeniala et al., 2009). Les caprins représentent plus de $85 \%$ du cheptel de petits ruminants (Rabeniala et al., 2009), c'est pourquoi ils ont été les seuls considérés dans ce travail. L'élevage caprin est orienté vers la production de viande et accessoirement vers la production de lait. La nutrition des caprins dans notre site d'étude est principalement constituée par des feuilles et des brindilles d'arbustes comme Commiphora sp., Rhigozum madagascariense, Talinella boiviniana, Dicoma incana, Diospyros latispathulata, Solanum bumeliaefolium et Chadsia flammea (Randriamalala, 2014). Les chevriers sortent les troupeaux caprins de leurs enclos vers 6-7 h du matin et les conduisent aux points d'eau les plus proches ( $<30$ minutes de marche) avant de les conduire aux pâturages où ils sont laissés sans attache pendant la journée. Les chevriers retournent aux pâturages pour ramener les troupeaux à l'enclos vers $15-16 \mathrm{~h}$. Le retour peut prendre plus de deux heures puisque les animaux continuent à se nourrir en chemin. Ainsi, la durée du parcours journalier est de 10-12 h (Randriamalala, 2014).

Dans le Sud-Ouest malgache, les saillies ont lieu pendant la saison des pluies, en janvier-février (figure 1). Les fourrages sont abondants dans le parcours naturel pendant cette période de l'année et les chèvres présentent un bon état d'embonpoint, ce qui les prédispose à la reproduction. La gravidité dure cinq à six mois et les mises bas ont lieu en début de saison sèche (mai-juin ; figure 1). Une faible disponibilité fourragère prévaut pendant cette saison et provoque un état général de maigreur chez les chèvres et de fortes mortalités chez les chevreaux nouveau-nés selon les éleveurs. Après une courte période de repos sexuel (> 40 jours), les femelles peuvent à nouveau être fécondées. Mais leur mauvais état d'embonpoint limite l'efficacité des accouplements et la proportion de chèvres gravides pendant la saison sèche est faible. Les mises bas correspondantes surviennent en saison des pluies (janvier-février ; figure 1), ce qui améliore le taux de survie de chevreaux. Les interventions liées au flushing ont eu pour but

\begin{tabular}{|c|c|c|c|c|c|}
\hline $\begin{array}{l}\text { Saillies } \\
\text { saison des } \\
\text { pluies }\end{array}$ & $\begin{array}{c}\text { Premières } \\
\text { mises bas } \\
\text { (saison sèche) }\end{array}$ & $\begin{array}{l}\text { Début flushing } \\
\text { (saison sèche) }\end{array}$ & $\begin{array}{l}\text { Saillies saison } \\
\text { sèche }\end{array}$ & $\begin{array}{l}\text { Fin flushing } \\
\text { (saison sèche) }\end{array}$ & $\begin{array}{c}\text { Secondes } \\
\text { mises bas } \\
\text { (saison des pluies) }\end{array}$ \\
\hline Janv-fév & $\begin{array}{c}\text { Mai-juin } \\
\text { J-85 }\end{array}$ & $\begin{array}{c}\mid \\
\text { Juil-août } \\
\text { J-30 }\end{array}$ & Août-Sept & $\begin{array}{l}\text { Août-Sept } \\
\mathrm{J}+15\end{array}$ & $\begin{array}{c}\mid \\
\text { Janv-fév } \\
J+150\end{array}$ \\
\hline
\end{tabular}

Figure 1 : chronogramme de la reproduction améliorée des chèvres du Sud-Ouest malgache. 
a) d'augmenter la proportion de chèvres fécondées en saison sèche dans les troupeaux (juin-juillet), et/ou b) de synchroniser les chaleurs des femelles reproductrices pendant cette saison et ainsi d'augmenter le nombre de mises bas en saison des pluies (janvier-février; figure 1).

\section{Protocole expérimental et traitement des données}

Onze éleveurs pilotes dans les deux communes rurales ont été sélectionnés, soit 391 chèvres dont 207 traitées et 184 témoins (tableau I). Les critères de choix de ces éleveurs ont été leur volonté de collaborer, la disponibilité d'un troupeau contenant au moins 20 femelles reproductrices en pleine carrière et la disponibilité de mâles pour assurer les montes (au moins un mâle pour 15 femelles). Le flushing a été pratiqué durant la saison sèche (juillet à août 2013) pendant laquelle prévalaient une faible disponibilité fourragère et un mauvais état corporel des femelles reproductrices (amaigrissement excessif). Le manioc a été utilisé comme complément alimentaire. Cet aliment énergétique est cultivé et disponible localement et son prix est abordable par rapport aux autres aliments énergétiques. Avec des chèvres cyclées, le traitement par flushing consiste en un apport de manioc sec concassé à raison de 500 grammes par tête par jour (environ 0,53 unité fourragère ; André, 1975) pendant 45 jours (figure 1). En effet, la durée minimale de l'intervalle entre mise bas et premières chaleurs est de 35-42 jours pour les chèvres (figure 1 ; Zarrouk et al., 2001). Ces 500 grammes apportent les suppléments d'énergie qui favorisent la reproduction, les besoins énergétiques pour l'entretien de l'animal étant apportés par les fourrages naturels au pâturage. Le manioc était proposé après l'abreuvement afin d'éviter d'éventuelles intoxications dues aux glycosides cyanogéniques.

Les comportements alimentaires journaliers des animaux, les parcours naturels ainsi que les modes de conduite des troupeaux n'ont pas été modifiés durant le traitement. En particulier, aucune séparation des boucs et des femelles dans le troupeau n'a été mise en place. Une nouvelle chèvrerie a été construite dans chaque élevage pour isoler les femelles traitées pendant l'alimentation qui n'a duré pas plus d'une heure par jour. Les troupeaux (traités et témoins) ont été déparasités par voie orale à l'aide de produits à large spectre à base d'ivermectine, de lévamizole ou d'albendazole sept jours avant le début du traitement, aux doses prescrites. Un rappel de déparasitage a eu lieu dix jours plus tard afin d'éviter la spoliation des éléments

\section{Tableau I}

Distribution des lots de chèvres dans le site d'étude (Sud-Ouest malgache)

\begin{tabular}{llccr} 
Commune & Village & Eleveur & \multicolumn{2}{c}{ Effectif } \\
\cline { 3 - 5 } & & & Traité & Témoin \\
\hline \multirow{3}{*}{ Soalara-Sud } & Soalara & E1 & 20 & 23 \\
& Antsirafaly & E2 & 20 & 5 \\
& Antsirafaly & E3 & 20 & 42 \\
& Antsirafaly & E4 & 20 & 11 \\
& Akaranila & E5 & 20 & 15 \\
& Akaranila & E6 & 20 & 18 \\
& Akaranila & E7 & 20 & 25 \\
Beheloka & Andranotohoke & E8 & 20 & 14 \\
& Manandro & E9 & 16 & 8 \\
& Manandro & E10 & 20 & 17 \\
& Andrakalily & E11 & 11 & 6 \\
\hline \multirow{2}{*}{ Total } & & & 207 & 184
\end{tabular}

nutritifs par les parasites intestinaux. Les performances de reproduction des femelles ont été caractérisées par les paramètres suivants : le taux de fertilité, qui est le rapport entre le nombre de femelles mettant bas et le nombre de femelles mises à la reproduction; le taux de fécondité, qui est le nombre de chevreaux nés rapporté au nombre de femelles mises à la reproduction ; le taux de prolificité, défini comme le nombre de chevreaux nés, ramené au nombre de mises bas ; et le taux de survie à un mois qui est le rapport entre le nombre de chevreaux survivants 30 jours après la naissance et le nombre de femelles mises à la reproduction.

Des tests de fréquences (Xlstat 6.03 ; Addinsoft, 1995-2008) ont été utilisés pour comparer les taux de fertilité, de fécondité et de survie des troupeaux traités et témoins. Des tests de comparaison de moyennes pour échantillons appariés (Xlstat 6.03) ont été appliqués pour analyser les effets du traitement sur les valeurs moyennes des paramètres de reproduction (taux de fertilité, de fécondité et de survie des troupeaux traités et témoins).

La rentabilité de la pratique du flushing a été calculée en comparant le coût de revient au chiffre d'affaires (CA). Le coût de revient de la pratique du flushing comprenait le coût du manioc sec et le coût des produits de déparasitage. Celui de la conduite d'élevage traditionnelle était pratiquement nul puisqu'aucun intrant n'a été utilisé. Le CA provenait des ventes d'animaux. Si l'on suppose que l'éleveur vend un nombre d'individus équivalent à celui de chevreaux nés dont la survie est assurée, alors le CA par femelle reproductrice peut se calculer selon l'équation (1) :

$\mathrm{CA}=$ Taux de fécondité $\times$ Taux de survie à 30 jours $\times$ Prix d'un animal adulte

Le bénéfice maximal espéré est la différence entre le CA et le coût de revient.

\section{RESULTATS}

\section{Fertilité,fécondité et survie à 30 jours}

Les taux moyens de fécondité et de fertilité des femelles traitées (respectivement $81 \%$ et $69 \%$ ) ont été globalement plus élevés que ceux des témoins (respectivement $28 \%$ et $25 \%$; tableau II ; figure 2). Le flushing a plus que doublé les taux de fertilité et de fécondité des chèvres. Les taux de fécondité des individus traités dans les villages de Soalara-Sud (E1 à E8) ont été particulièrement élevés et ont été significativement différents de ceux des individus témoins des mêmes villages ( $p \leq 0,004$; tableau II). Le taux moyen de survie à 30 jours des chevreaux des femelles traitées a également été significativement plus élevé que celui des témoins (67\% vs $258 \%$; tableau II ; figure 2).

\section{Prolificité}

Le taux de prolificité des troupeaux traités a été proche de $100 \%$ (117\% ; $\mathrm{n}=207$; tableau II ; figure 2), ce qui correspond à des naissances simples, comparables à celles des témoins $(106 \% ; \mathrm{n}=184)$. La supplémentation alimentaire n'a pas eu d'effet significatif sur le nombre de chevreaux nés par portée.

\section{Rentabilité potentielle de la pratique du flushing}

Le coût du manioc sec était environ de 2,50 euros (€) par tête et celui des produits de déparasitage environ de $0,13 €$. Le coût de revient du flushing était ainsi environ de 2,6€. Le prix minimal d'une chèvre adulte sur le marché était environ de 12,50 € en 2013-2014. En appliquant l'équation (1), le CA associé à une chèvre adulte traitée pendant une saison sèche était de $6,78 €$ (tableau III). Le bénéfice de l'éleveur par tête et pour six mois était ainsi de $4,15 €$ pour une chèvre traitée. Le revenu maximal espéré provenant de la pratique du flushing était 


\section{Tableau II}

Résultats des essais sur la reproduction sur des chèvres du Sud-Ouest malgache

\begin{tabular}{|c|c|c|c|c|c|c|c|c|c|c|c|c|c|c|}
\hline Eleveur & & E1 & E3 & E4 & E5 & E6 & E7 & E8 & E9 & E10 & E11 & E12 & Total & Moy. \\
\hline \multirow[t]{2}{*}{$\mathrm{N}$ total } & $\mathrm{T}$ & 20 & 20 & 20 & 20 & 20 & 20 & 20 & 20 & 16 & 20 & 11 & 207 & \\
\hline & t & 23 & 5 & 42 & 11 & 15 & 18 & 25 & 14 & 8 & 17 & 6 & 184 & \\
\hline \multirow[t]{2}{*}{ Naissance } & $\mathrm{T}$ & 12 & 20 & 18 & 16 & 15 & 20 & 12 & 5 & 12 & 14 & 3 & 147 & \\
\hline & $\mathrm{t}$ & 4 & 1 & 17 & 3 & 2 & 7 & 6 & 0 & 5 & 6 & 0 & 51 & \\
\hline \multirow[t]{2}{*}{ Chevreau né } & $\mathrm{T}$ & 12 & 24 & 18 & 16 & 24 & 23 & 17 & 9 & 12 & 14 & 3 & 172 & \\
\hline & $\mathrm{t}$ & 4 & 2 & 17 & 3 & 2 & 7 & 8 & 0 & 5 & 6 & 0 & 54 & \\
\hline \multirow{2}{*}{$\begin{array}{l}\text { Survivant } \\
\text { après } 30 \text { jours }\end{array}$} & $\mathrm{T}$ & 12 & 20 & 18 & 16 & 14 & 16 & 12 & 5 & 12 & 14 & 3 & 142 & \\
\hline & $t$ & 4 & 1 & 17 & 3 & 2 & 7 & 6 & 0 & 5 & 6 & 0 & 51 & \\
\hline \multirow{4}{*}{$\begin{array}{l}\text { Taux de } \\
\text { fécondité (\%) }\end{array}$} & $\mathrm{T}$ & 60 & 120 & 90 & 80 & 120 & 115 & 85 & 45 & 75 & 70 & 27 & 83 & $81(20)$ \\
\hline & $t$ & 17 & 40 & 40 & 27 & 13 & 39 & 32 & 0 & 63 & 35 & 0 & 29 & $28(13)$ \\
\hline & $P$ & 0,004 & $<0,001$ & $<0,001$ & 0,004 & $<0,001$ & $<0,001$ & $<0,001$ & 0,003 & 0,525 & 0,035 & 0,159 & $<0,001$ & \\
\hline & z & 2,88 & 3,69 & 3,68 & 2,88 & 5,25 & 4,15 & 3,56 & 2,93 & 0,64 & 2,11 & 1,41 & 10,74 & \\
\hline \multirow{4}{*}{$\begin{array}{l}\text { Taux de } \\
\text { fertilité (\%) }\end{array}$} & $\mathrm{T}$ & 60 & 100 & 90 & 80 & 75 & 100 & 60 & 25 & 75 & 70 & 27 & 71 & $69(17)$ \\
\hline & $t$ & 17 & 20 & 40 & 27 & 13 & 39 & 24 & 0 & 63 & 35 & 0 & 28 & $25(12)$ \\
\hline & p & 0,004 & $<0,001$ & $<0,001$ & 0,004 & $<0,001$ & 0,01 & 0,014 & 0,043 & 0,525 & 0,035 & 0,159 & $<0,001$ & \\
\hline & z & 2,88 & 4,36 & 3,68 & 2,88 & 3,33 & 2,59 & 2,45 & 2,03 & 0,64 & 2,11 & 1,41 & 8,07 & \\
\hline \multirow{2}{*}{$\begin{array}{l}\text { Taux de } \\
\text { prolificité (\%) }\end{array}$} & $\mathrm{T}$ & 100 & 120 & 100 & 100 & 160 & 115 & 142 & 180 & 100 & 100 & 100 & 117 & $120(19)$ \\
\hline & $\mathrm{t}$ & 100 & 200 & 100 & 100 & 100 & 100 & 133 & . & 100 & 100 & & 106 & $115(27)$ \\
\hline \multirow{4}{*}{$\begin{array}{l}\text { Taux de survie } \\
\text { à } 30 \text { jours }(\%)\end{array}$} & $\mathrm{T}$ & 60 & 100 & 90 & 80 & 70 & 80 & 60 & 25 & 75 & 70 & 27 & 69 & $67(16)$ \\
\hline & $t$ & 17 & 20 & 40 & 27 & 13 & 39 & 24 & 0 & 63 & 35 & 0 & 28 & $25(12)$ \\
\hline & p & 0,004 & $<0,001$ & $<0,001$ & 0,004 & 0,001 & 0,01 & 0,014 & 0,043 & 0,525 & 0,035 & 0,159 & $<0,001$ & \\
\hline & z & 2,88 & 4,36 & 3,68 & 2,88 & 3,33 & 2,59 & 2,45 & 2,03 & 0,64 & 2,11 & 1,41 & 8,55 & \\
\hline
\end{tabular}

Moy. : moyenne ; $\mathrm{T}$ : traité ; $\mathrm{t}$ : témoin ; $\mathrm{p}$ : degré de signification des tests de fréquences ; $\mathrm{z}$ : statistique des tests de fréquences ; (entre parenthèses, dernière colonne) : erreur standard
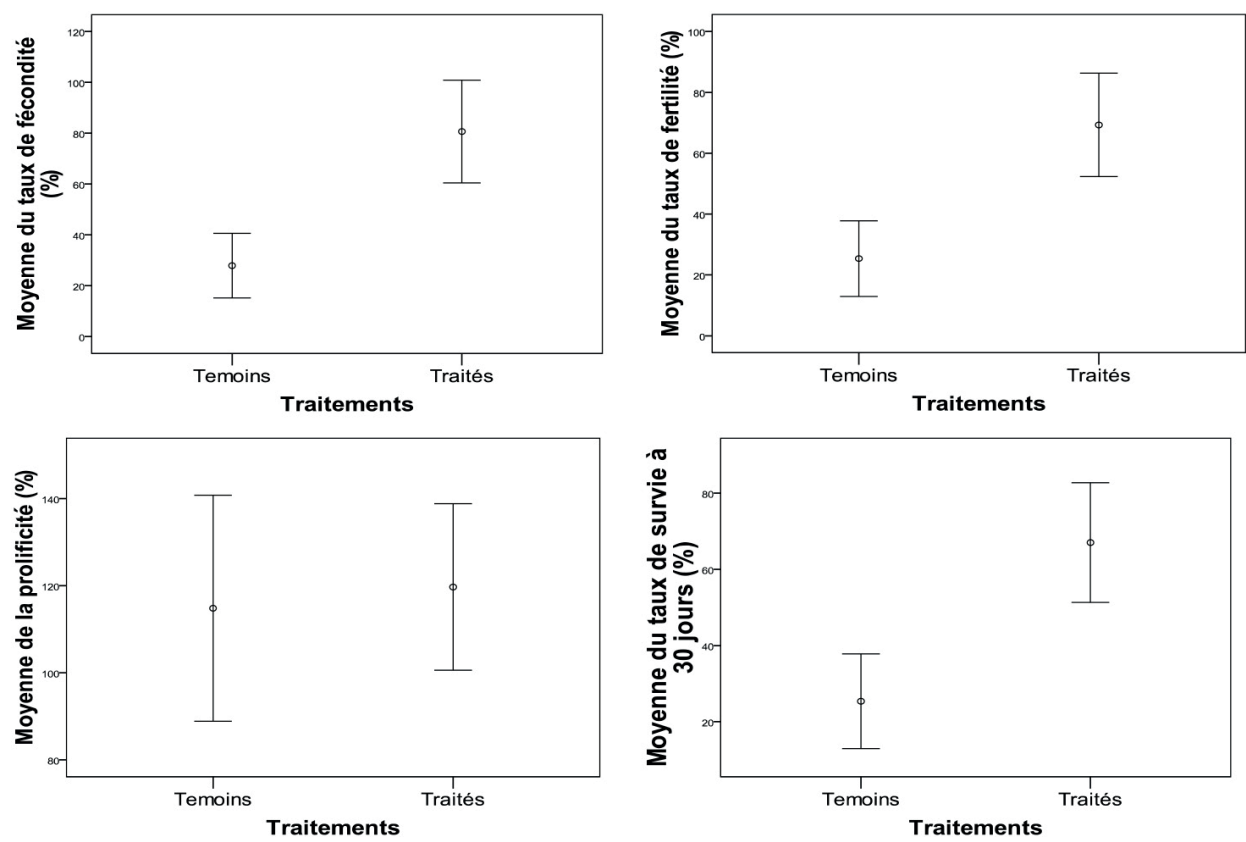

Figure 2 : effets globaux des traitements sur les paramètres de reproduction des chèvres du SudOuest malgache; barre verticale : erreur standard. 
largement supérieur à celui obtenu par l'élevage traditionnel $(0,91 €$; tableau III).

Le tableau IV résume les résultats sur la reproduction des caprins en les comparant avec la littérature où diverses méthodes de groupage des chaleurs ont été utilisées.

\section{DISCUSSION}

\section{Limite et avantage de l'approche adoptée}

L'étude a été menée chez des éleveurs autochtones. Des facteurs de la reproduction n'ont pas été maîtrisés : les moments de l'ovulation, des chaleurs et éventuellement de l'accouplement. Ce n'est pas le cas pour la plupart des études sur la synchronisation de l'œstrus chez les chèvres, menées dans des sites d'expérimentation où les paramètres de reproduction comme les moments d'ovulation, des chaleurs, d'accouplement, de gravidité et de mise bas sont maîtrisés (Regueiro et al., 1999 ; Motlomelo et al., 2002 ; Lehloenya et Greyling, 2010). Par contre, les présents résultats ont l'avantage d'être plus réalistes et plus faciles à vulgariser car ils n'ont pas été obtenus dans les conditions optimales des sites d'expérimentation, mais dans les conditions réelles de l'élevage. De plus, les naissances en janvier des chevreaux issus des chaleurs induites en saison sèche par le flushing coïncident avec le début de la saison des pluies, période d'abondance de fourrages, ce qui laisse présager un faible taux des mortalités liées à l'insuffisance alimentaire. En effet, les saillies habituelles des chèvres dans le site d'étude ont lieu en janvier-février et les naissances correspondantes se produisent en mai-juillet, pendant la saison sèche au cours de laquelle prévaut une faible disponibilité fourragère, ce qui contribue à un taux élevé de mortalités chez les nouveau-nés, selon les éleveurs.

\section{Comparaison avec d'autres techniques de synchronisation des chaleurs}

Au Zimbabwe, Kusina et al. (2001) ont effectué des recherches sur les effets de différents niveaux d'énergie correspondant à l'alimentation de chèvres Mansona sur la synchronisation de leurs chaleurs et sur leur fertilité en utilisant la monte naturelle et deux injections, espacées de 11 jours, de $100 \mathrm{mg}$ de cloprostenol. Ils trouvent des taux moyens de fertilité globalement supérieurs (73-100\%) à celui des chèvres traitées dans notre étude (69\%). Il en est de même avec les taux moyens de fécondité de $120-160 \%$ contre $81 \%$ dans notre étude. De même, l'utilisation d'éponges imbibées de $60 \mathrm{mg}$ d'acétate de médroxyprogestérone et de l'effet mâle sur des chèvres se nourrissant d'un pâturage à Sorghum vulgare, Medicago sativa et Sesbania aculeata, (Kausar et al., 2009) au Pakistan a abouti à l'obtention d'un taux moyen de fertilité de $87,5 \%$, plus élevé que celui de notre étude $(69 \%)$. Par contre, la moyenne des taux de prolificité obtenus dans leur étude (130\%) est comparable à celle de notre étude (120\%). Enfin, l'usage d'hormone gonadotrophine chorionique équine (eCG) ou de la supplémentation alimentaire (orge, huile de soja ou de tournesol) couplé avec des inséminations artificielles sur des chèvres en Iran ont abouti à des taux moyens de fertilité de $88-100 \%$ (Kia et al., 2012), nettement supérieurs à celui de nos chèvres traitées. Les taux

\section{Tableau III}

Rentabilité potentielle de la pratique du flushing dans les troupeaux de chèvres du Sud-Ouest malgache

\begin{tabular}{cccccr}
$\begin{array}{l}\text { Nb. de chèvres mises à } \\
\text { la reproduction }\end{array}$ & $\begin{array}{c}\text { Prix d'un animal } \\
\text { adulte }(\boldsymbol{€})\end{array}$ & $\begin{array}{c}\text { Chiffre d'affaires } \\
\text { avec flushing }(€)\end{array}$ & $\begin{array}{c}\text { Coût de revient } \\
\text { flushing }(€)\end{array}$ & $\begin{array}{c}\text { Bénéfice } \\
\text { flushing }(€)\end{array}$ & $\begin{array}{r}\text { Chiffre d'affaires } \\
\text { sans flushing }(€)\end{array}$ \\
\hline 1 & 12,50 & 6,78 & 2,63 & 4,15 & 0,91 \\
10 & 12,50 & 67,84 & 26,25 & 41,59 & 9,10 \\
20 & 12,50 & 135,68 & 52,50 & 83,18 & 18,20 \\
30 & 12,50 & 203,51 & 78,75 & 124,76 & 27,30 \\
40 & 12,50 & 271,35 & 105,00 & 166,35 & 36,40 \\
60 & 12,50 & 407,03 & 157,50 & 249,53 & 54,60
\end{tabular}

\section{Tableau IV}

Paramètres de reproduction des caprins résultant de diverses méthodes de groupage des chaleurs (valeurs moyennes) dans le monde

\begin{tabular}{|c|c|c|c|c|}
\hline \multirow[t]{2}{*}{ Auteur } & \multirow[t]{2}{*}{ Méthode } & \multicolumn{3}{|c|}{ Paramètre de reproduction } \\
\hline & & $\begin{array}{c}\text { Taux de } \\
\text { fertilité (\%) }\end{array}$ & $\begin{array}{c}\text { Taux de } \\
\text { fécondité (\%) }\end{array}$ & $\begin{array}{c}\text { Taux de } \\
\text { prolificité (\%) }\end{array}$ \\
\hline Présente étude & $\begin{array}{l}\text { Supplémentation alimentaire manioc séché + monte } \\
\text { naturelle }\end{array}$ & 69 & 81 & 120 \\
\hline Kusina et al. (2001) & $\begin{array}{l}\text { Supplémentation alimentaire à niveaux variables d'énergie + } \\
\text { monte naturelle }\end{array}$ & $73-100$ & $120-160$ & $90-160$ \\
\hline Kausar et al. (2009) & Hormone + effet mâle + monte naturelle & 87 & - & 130 \\
\hline \multirow[t]{2}{*}{ Kia et al. (2012) } & Hormone + insémination artificielle & $88-100$ & - & $157-200$ \\
\hline & $\begin{array}{l}\text { Supplémentation alimentaire avec de l'orge, de l'huile de } \\
\text { soja et de tournesol + insémination artificielle }\end{array}$ & 88-100 & - & $157-179$ \\
\hline \multirow{2}{*}{$\begin{array}{l}\text { Santiago-Miramontes } \\
\text { et al. (2012) }\end{array}$} & Hormone + supplémentation alimentaire + monte naturelle & $35-55$ & - & $140-190$ \\
\hline & Supplémentation alimentaire + monte naturelle & 40 & - & 150 \\
\hline
\end{tabular}


moyens de prolificité correspondants (157-200\% ; Kia et al., 2012) sont également plus importants que celui que nous avons obtenu $(120 \%)$.

Le taux moyen de fertilité des animaux traités dans le Sud-Ouest malgache a été cependant supérieur à ceux de 35-55 \% de SantiagoMiramontes et al. (2011). Ces derniers ont testé les effets de suppléments alimentaires et d'hormone sur la synchronisation et les paramètres de reproduction de chèvres au Mexique ; ils ont également trouvé des taux moyens de prolificité (140-190\%) supérieurs à celui de nos chèvres traitées $(120 \%)$.

\section{Méthode efficace et applicable dans le contexte du Sud malgache}

L'application de la supplémentation alimentaire a globalement amélioré les paramètres de reproduction des individus traités et est potentiellement rentable. Les méthodes utilisées sont par contre relativement simples et modifient peu les pratiques pastorales existantes. La supplémentation alimentaire permet ainsi d'augmenter le nombre de naissances (au moins trois mises bas par chèvre par période de deux ans), de décaler les naissances en saison humide et d'assurer ainsi un meilleur taux de survie des chevreaux. Dans tous les cas, une augmentation significative de la production de l'élevage caprin résulterait de l'adoption de cette technique par les éleveurs, ce qui risquerait de conduire à un surpâturage si les surplus d'animaux produits n'étaient pas commercialisés. La maîtrise de la reproduction par l'intermédiaire de la supplémentation alimentaire peut rentrer dans le cadre de la conduite d'élevage en lots qui consiste à produire périodiquement des lots d'animaux d'âges voisins par le groupage des chaleurs. Les animaux obtenus assurent la pérennité du cheptel par le renouvellement des reproducteurs.

La vente du surplus d'animaux, ceux dans les classes d'âge supérieures, permet de maintenir la taille du troupeau, d'utiliser durablement les ressources naturelles (pâturages et espèces fourragères) par le contrôle de la charge et de générer des revenus supplémentaires pour l'éleveur. Les débouchés pour absorber ces surplus d'animaux ne devraient pas manquer. En effet, l'effectif des zébus à Madagascar a connu une baisse durant les 40 dernières années (12 millions en 1970 et 10 millions en 2004 ; ministère de l'Elevage, 2012) alors que leur viande constitue la première source de protéines animales dans le pays. La hausse de la demande liée à la croissance démographique, l'exportation, la raréfaction des reproducteurs suite au vol de bovidés dans les zones naisseuses et les problèmes sanitaires sont les principales causes de cette baisse de l'effectif. Par conséquent, l'offre en viande rouge régresse avec cette baisse de l'effectif bovin alors que la demande augmente avec la population. Pour atténuer ce déséquilibre, les petits ruminants, dont les caprins, sont appelés à devenir une source alternative de viande rouge à Madagascar. De plus, les demandes en viande de petits ruminants de la part de pays musulmans comme les Comores et les pays du golfe Persique comme l'Arabie Saoudite, et d'autres pays comme la Chine, n'arrivent pas à être satisfaites pour le moment, faute de production suffisante et de la mauvaise organisation de la filière petits ruminants (obs. pers.).

Par ailleurs, une attention particulière doit être portée aux éventuels effets néfastes du pâturage des caprins sur la régénération des fourrés xérophiles comme c'est le cas dans certaines formations sèches en Namibie (Moser-Norgaard et Denich, 2011) ou au nord-ouest de la Chine (Saumel et al., 2011). Une évaluation plus précise des capacités de charge des pâturages existants et des simulations des effets de l'adoption de la supplémentation alimentaire sur les effectifs caprins doivent être faites. En effet, les effets négatifs du pâturage des caprins sur la végétation des zones semi-arides sont principalement des conséquences de charges animales trop élevées (Rosa-Garcia et al., 2012 ; Randriamalala et al., 2016). Ce problème peut être résolu en partie par la pratique de la conduite d'élevage en lots qui augmente les revenus des éleveurs tout en maintenant constante la taille du cheptel par la vente régulière d'animaux. De plus, la pratique d'une gestion appropriée des pâturages comme leur rotation peut diminuer considérablement la pression des caprins sur le pâturage et peut être bénéfique à la conservation de la biodiversité dans le site d'étude (Randriamalala et al., 2016).

\section{CONCLUSION}

Le flushing est une méthode simple et efficace pour grouper les chaleurs des caprins du Sud-Ouest malgache en vue de concentrer les naissances en saison de pluie pour augmenter le nombre de naissances ( $\geq 3$ tous les deux ans) et le taux de survie des chevreaux. La vente des surplus d'animaux provenant de l'adoption de cette technique de maîtrise de la reproduction peut être une source importante de revenu pour les éleveurs, surtout si elle rentre dans le cadre d'une conduite d'élevage en lots qui modifie peu les pratiques pastorales et qui est ainsi plus facile à vulgariser. Des travaux importants de sensibilisation et de formation des éleveurs sur la conduite d'élevage en lots doivent être préalablement entrepris avant la vulgarisation du flushing.

\section{Remerciements}

Nous remercions le programme Eastern and Southern Africa Partnership Programme (ESAPP) du Centre of Development and Environment (CDE ; Université de Berne, Suisse) qui a financé ce travail par les projets Q097 et Q918. Nous remercions aussi chaleureusement les éleveurs qui ont accepté de collaborer avec nous.

\section{Déclaration des contributions des auteurs}

JHA et JRR ont conçu l'étude ; JHA l'a planifiée ; JHA et RR ont collecté les données ; JHA, JRR, AJMR, RR les ont interprétées ; $\mathrm{JRR}$ a rédigé la première version du manuscrit ; tous les auteurs ont fait des révisions critiques du manuscrit et ont donné l'accord final de la version à publier

\section{Conflits d'intérêts}

Létude a été réalisée sans conflit d'intérêts.

\section{REFERENCES}

Acero-Camelo A., Valencia E., Rodríguez A., Randel P.-F., 2008. Effects of flushing with two energy levels on goat reproductive performance. Livest. Res. Rural Dev., 20 (9)

Amoah E.-A., Gelaye S., Guthrie P., Rexroad Jr C.-E., 1996. Breeding season and aspects of reproduction of female goats. J. Anim. Sci., 74: 723-728, doi : $10.2527 / 1996.744723 x$

André C., 1975. L'alimentation des animaux domestiques à Madagascar : Les aliments-le rationnement. Service de l'enseignement et de la formation agricole, Madagascar

Andrianarisoa J.-H., 2013. Supplémentation alimentaire pour améliorer la performance de reproduction des caprins; Cas de la commune rurale de Soalara Sud- District de Toliara II. Mém. DEA ESSA-Forêts, Université d'Antananarivo, Madagascar

Chemineau P., 1989. L'effet bouc: mode d'action et efficacité pour stimuler la reproduction des chèvres en anœstrus. Prod. Anim., 2 (2) : 97-105

Cornet A., Guillaumet J.-L., 1976. Divisions floristiques et étages de végétation à Madagascar. Cah. ORSTOM, Sér. Biol., $9: 35-42$

Feldt T., Neudert R., Fust P., Schlect E., 2016. Reproductive and economic performance of local livestock in southwestern Madagascar: Potentials and constraints of a highly extensive system. Agr. Syst., 149: 54-64, doi: 10.1016/j.agsy.2016.08.007 
Gaspard D., Venegas-González A., Beeckman H., Randriamalala R.-J., De Ridder M., Tomazello-Filho M., Ramananantoandro T., 2018. Tree ring responses to climate variability from xerophytic thicket of South Soalara, Madagascar. Dendrochronologia, 49: 57-67, doi: 10.1016/j.dendro.2018.02.003

Greyling J.-P.-C., Van der Nest M., 2000. Synchronization of oestrus in goats: dose effect of progestagen. Small Rumin. Res., 36: 201-207, doi: 10.1016/ S0921-4488(99)00165-0

Hanke H., 2016. Livelihoods on the edge: Farming household income, food security and resilience in Southwestern Madagascar. Mém. Doct. Université de Gottingen, Allemagne

Kausar R., Khanum S.-A., Hussain M., Shah M.-S., 2009. Estrus synchronization with medroxyprogestérone acetate impregnated sponges in goats (Capra hircus). Pak. Vet. J., 29 (1): 16-18

Kia H.-D., Chapdareh W.-M., Khani A.-H., Moghaddam G., Rashidi A., Sadri H., Alijani S., 2012. Effects of flushing and hormonal treatment on reproductive performance of Iranian Markhoz goats. J. Anim. Physiol. An. N., 96: 1157-1164, doi: 10.1111/j.1439-0396.2011.01234.x

Kusina N.-T., Chinuwo T., Hamudikuwanda H., Ndlovu L.-R., Muzanenhamo S., 2001. Effect of different dietary energy level intakes on efficiency of estrus synchronization and fertility in Mashona goat does. Small Rumin. Res., 39: 283-288, doi: 10.1016/S0921-4488(00)00192-9

Lehloenya K.-C., Greyling J.-P.-C., 2010. The ovarian response and embryo recovery rate in Boer goat does following different superovulation protocols, during the breeding season. Small Rumin. Res., 88: 38-43, doi: 10.1016/j.smallrumres.2009.11.007

Lehloenya K.-C., Greyling J.-P.-C., Grobler S, 2008. Effect of season on the superovulatory response in Boer goat does. Small Rumin. Res., 78: 74-79, doi: 10.1016/j.smallrumres.2008.05.003

Lehloenya K.-C., Greyling J.-P.-C., Schwalbach L.-M.-J., 2005. Reproductive performance of South African indigenous goats following oestrous synchronisation and artificial insemination. Small Rumin. Res., 57: 115-120, doi: 10.1016/j.smallrumres.2004.05.004

Ministère de l'élevage, 2012. Données statistiques. SAIGS/DSI. Antananarivo, Madagascar

Molle G., Branca A., Ligios S., Sitzia M., Casu S., Landau S., Zoref Z., 1995. Effect of grazing background and flushing supplementation on reproductive performance in Sarda ewes. Small Rumin. Res., 17: 245-254, doi: 10.1016/0921-4488(95)00691-D

Molle G., Landau S., Branca A., Sitzia M., Fois N., Ligios S., Casu S., 1997. Flushing with soybean meal can improve reproductive performances in lactating Sarda ewes on a mature pasture. Small Rumin. Res., 24: 157-165, doi: 10.1016/S0921-4488(96)00950-9
Moser-Norgaard P.-M., Denich M., 2011. Influence of livestock on the regeneration of fodder trees along ephemeral rivers of Namibia. J. Arid Environ., 75: 371-376, doi: 10.1016/j.jaridenv.2010.11.009

Motlomelo K.-C., Greyling J.-P.-C., Schwalbach L.-M.-J., 2002. Synchronisation of oestrus in goats: the use of different progestagen treatments. Small Rumin. Res., 45: 45-49, doi: 10.1016/S0921-4488(02)00113-X

Rabeniala R., Raoliarivelo L.-I.-B., Masezamana H.-N., Andrianarisoa J.-H., Randriamalala R.-J., 2009. Gestion de pâturage pour le cheptel de petits ruminants (ovins et caprins) dans une zone semi-aride du district de toliara II. Rapport final projet ESAPP Q906, DERAD, Antananarivo, Madagascar

Randriamalala, H., 2014. Etude des comportements alimentaires des caprins en zone semi aride de Madagascar, cas de la commune rurale de Soalara Sud. Mém. DEA, ESSA-Forêts, Université d'Antananarivo, Madagascar

Randriamalala R.-J., Radosy H.-O., Razanaka S., Randriambanona H., Hervé D., 2016. Effects of goat grazing and woody charcoal production on xerophytic thickets of southwestern Madagascar. J. Arid Environ., 128: 65-72, doi: 10.1016/j.jaridenv.2016.01.002

Randriamalala R.-J., Ramananantoandro T., Radosy H.-O., Randriambanona H., Hervé D., 2017. Annual biomass increment of xerophytic thickets and sustainability of woody charcoal production in southwestern Madagascar. For. Ecol. Manag., 400: 139-149, doi: 10.1016/j.foreco.2017.05.049

Raoliarivelo L.-I.-B., Rabeniala R., Masezamana H.-N., Andrianarisoa J.-H., Randriamalala R.-J., 2010. Impact de la fabrication de charbon de bois sur la production et la disponibilité fourragère de pâturage en zone subaride, cas de la commune de Soalara-Sud, Toliara II. Rapport final projet ESAPP Q909, DERAD, Antananarivo, Madagascar

Regueiro M., Pérez Clariget R., Ganzabal A., Aba M., Forsberg M., 1999 Effect of medroxyprogesterone acetate and eCG treatment on the reproductive performance of dairy goats. Small Rumin. Res., 33: 223-230, doi: 10.1016/S0921-4488(99)00024-3

Rosa-García R., Celaya R., García U., Osoro K., 2012. Goat grazing, its interactions with other herbivores and biodiversity conservation issues. Small Rumin. Res., 107: 49-64, doi: 10.1016/j.smallrumres.2012.03.021

Santiago-Miramontes M.-A., Luna-Orozco J.-R., Meza-Herrera C.-A., Rivas-Muñoz R., Carrillo E., Véliz-Deras F.-G., Mellado M., 2011. The effect of flushing and stimulus of estrogenized does on reproductive performance of anovulatory-range goats. Trop. Anim. Health Prod., 43: 1595 1600, doi: 10.1007/s11250-011-9849-6

Saumel I., Ziche D., Yub R., Kowarik I., Overdieck D., 2011. Grazing as driver for Populus euphratica woodland degradation in the semi-arid Aibi Hu region, northwestern China. J. Arid Environ., 75: 265-269, doi: 10.1016/j.jaridenv.2010.10.013

Zarrouk A., Souilem O., Drion P.-V., Beckers J.-F., 2001. Caractéristiques de la reproduction de l'espèce caprine. Ann. Méd. Vét., 145 : 98-105

\section{Summary}

Andrianarisoa J.H., Randriamalala J.R., Mbolatianarizao Randrianariveloseheno A.J., Rabeniala R. Feed supplementation to synchronize estrous and improve the reproductive performance of goats in Madagascar

The coastal areas of Southwestern Madagascar are the driest on the island. The breeding of small ruminants is the main source of income for the local population. However, their reproduction, especially that of goats, is hampered by unfavorable zootechnical (genetics and animal husbandry) and agroclimatic conditions (low fodder availability during the long dry season). Methods to improve the reproductive performance of goat breeding exist: artificial insemination, hormonal treatments, male effect and feed supplementation or flushing. Flushing is a simple method to implement and does not require any major change in pastoral practices; it is best

\section{Resumen}

Andrianarisoa J.H., Randriamalala J.R., Mbolatianarizao Randrianariveloseheno A.J., Rabeniala R. Suplementación alimenticia para sincronizar los calores y mejorar los rendimientos reproductivos de los caprinos en Madagascar

Las zonas litorales del sudoeste malgache son las más áridas de la isla. La cría de pequeños rumiantes es la principal fuente de ingresos para las poblaciones locales. Sin embargo, su producción, sobre todo la de caprinos, se encuentra bloqueada por condiciones zootécnicas desfavorables (genética y prácticas de crianza) y agroclimas (baja disponibilidad forrajera durante la larga estación seca). Existen métodos de mejoramiento de los rendimientos reproductivos en la cría caprina: la inseminación artificial, los tratamientos hormonales, el efecto macho y la suplementación alimenticia o flushing. El flushing es un método simple de implementar y no implica 
adapted to the conditions of this semi-arid site. Two groups of animals treated against parasite were used during 45 days in July-August (dry season): a group of 207 females given an energy feed (flushing) of dry cassava at 500 g/day, and a control group of 184 goats. Reproduction parameters were recorded: fertility rate, conception rate, prolificity rate and survival rate at 30 days of age. Significant improvements in the fertility ( $81 \%$ vs $28 \%$ ) and conception (69\% vs $25 \%$ ) of treated females were observed. The prolificity of the treated group $(120 \%)$ was slightly higher than that of the control group $(115 \%)$, with no significant difference. Thirty days after birth the kid survival rate in the treated group was significantly higher (67\% vs $25 \%$ in control). The flushing method improved the reproduction parameters of native goats as well as the numerical and economic productivity of goat rearing in the semi-arid zone of Southwestern Madagascar.

Keywords: goats, fertility, conception rate, flushing, Madagascar ninguna modificación importante de las prácticas pastoriles; fue el más adaptado a las condiciones de este sitio semiárido. Dos lotes de animales desparasitados se utilizaron durante 45 días en julio y agosto (estación seca): un lote de 207 hembras con un alimento energético (flushing), mandioca seca, en cantidad de 500 g/día, y un lote control de 184 cabras. Se registraron los parámetros reproductivos: tasa de fertilidad, tasa de fecundidad, tasa de prolificidad y tasa de sobrevida a la edad de 30 días. Se observaron mejorías significativas de la fertilidad ( $81 \%$ vs $28 \%$ ) y de la fecundidad (69\% vs $25 \%$ ) de las hembras tratadas. La prolificidad del lote tratado (120\%) fue ligeramente superior a la del lote control (115\%), sin diferencia significativa. Treinta días después del nacimiento, la tasa de sobrevida de los cabritos del lote tratado fue significativamente más elevada ( $67 \%$ vs $25 \%$ para los controles). El método del flushing mejoró los parámetros de reproducción de los caprinos autóctonos, así como la productividad numérica y económica de la cría caprina en zona semiárida del sudoeste malgache.

Palabras clave: caprino, fertilidad, prolificidad, flushing, Madagascar 